\title{
Differenzialdiagnose und -therapie pulmonaler Erkrankungen mit Angiitis und Granulomatose
}

\author{
Differential Diagnosis and Therapy of Pulmonary Diseases \\ with Angiitis and Granulomatosis
}

D. Frechen, J. Smith, C. Cornelissen, S. Kweider, S. Krüger

Medizinische Klinik I, Universitätsklinikum, RWTH Aachen

Seltene Erkrankungen stellen den Arzt immer vor eine Herausforderung. Auf pneumologischem Gebiet sind dies zum Beispiel Formen von pulmonaler Angiitis und Granulomatose. In dieser CMEFortbildung werden fünf Erkrankungen vorgestellt, die sich pulmonal manifestieren und deren histologische Gemeinsamkeit das Auftreten von Angiitis und Granulomen ist: lymphomatoide Granulomatose, bronchozentrische Granulomatose, nekrotisierende sarkoide Granulomatose, Wegener'sche Granulomatose und das ChurgStrauss-Syndrom. Beispielhaft zeigt $\bullet$ Abb. 1 das Lungengewebe einer Patientin mit einer nekrotisierenden sarkoiden Granulomatose mit Angiits und einer perivaskulären granulomatösen Entzündung.

Häufig führt die weitere Abklärung von unspezifischen Symptomen wie Dyspnoe, thorakalen Schmerzen, Husten, Fieber oder Hämoptysen zur Diagnose einer dieser Erkrankungen. Radiologisch zeigen sich bei pulmonalen Vaskulitiden multiple pulmonale Rundherde oder Infiltrationen, zum Teil auch mit Kavernenbildung. Differenzialdiagnostisch kommen infektiöse Erkrankungen, septisch-embolische Abszesse, multiple pulmonale Infarkte, Lymphome, andere Systemerkrankungen wie die Sarkoidose oder ein metas-

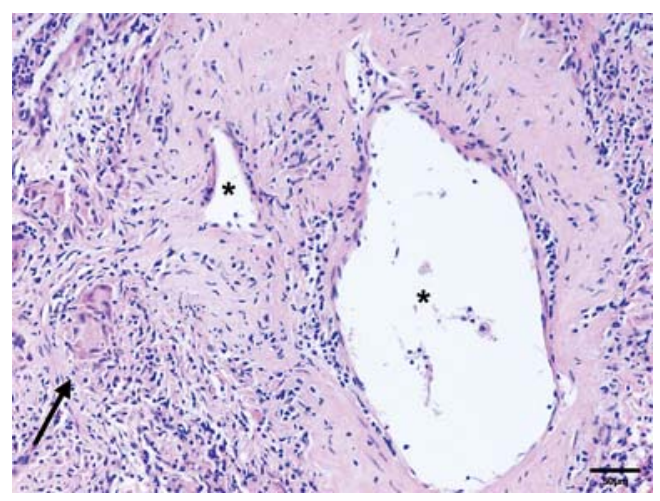

Abb. 1 Lungenbiopsie einer Patientin mit nekrotisierender sarkoider Ganulomatose (Hämatoxylin-Eosin, 20-fach): Nachweis von perivaskulärem Granulom mit mehrkernigen Riesenzellen vom Langhanstyp (Pfeil) und Angiitis ( ${ }^{*}$ ). tasierendes Karzinom in Betracht. Eine Übersicht über pulmonal manifestierende Erkrankungen, die mit einer Angiitis oder Granulomen einhergehen, gibt Tab. 1. Trotz der histologischen Gemeinsamkeit der fünf in diesem Artikel dargestellten Erkrankungen sind die krankheitstypischen Manifestationen, die Prognose und die Therapie unterschiedlich.

\section{Lernziele}

$\nabla$

Dieser CME-Beitrag soll eine Übersicht über pulmonal manifestierende Erkrankungen geben, die histologisch eine Angiitis und Granulome zeigen. Die differenzialdiagnostische Einordnung des histologischen Befundes kann erst durch weitere, die zugrunde liegende Erkrankung charakterisierende Befunde erfolgen und ist Voraussetzung für eine krankheitsspezifische Therapie.

\section{Geschichte}

\section{$\nabla$}

Nachdem Friedrich Wegener in den späten 30erJahren ein Syndrom aus systemischer Vaskulitis mit nekrotisierender granulomatöser Inflammation des Respirationstraktes und fokaler Glomerulitis beschrieb, charakterisierten Churg und Strauss 1951 ein Syndrom mit allergischer Angiitis und Granulomatose, welches später nach ihnen benannt wurde: das Churg-Strauss-Syndrom. 1972 unterteilte Liebow Erkrankungen mit pulmonaler Angiitis und Granulomatose aufgrund pathologischer Befunde und klinischer Manifestation in: Wegener'sche Granulomatose, lymphomatoide Granulomatose, nekrotisierende sarkoide Granulomatose und bronchozentrische Granulomatose [1].
VNR

2760512011060001745

Bibliografie

Dol http://dx.doi.org/

10.1055/s-0030-1256896

Pneumologie 2011; 65: 675-684

(c) Georg Thieme Verlag KG

Stuttgart · New York

ISSN 0934-8387

Korrespondenzadresse

Dr. med. Dirk Frechen

Medizinische Klinik I

Universitätsklinikum der RWTH

Aachen

Pauwelsstr. 30

52074 Aachen

dfrechen@ukaachen.de 
(CVP) oder Cylophosphamid, Doxorubicin, Vincristin, Prednison (CHOP). CVP und CHOP werden in Abhängigkeit vom Allgemeinzustand des Patienten mit Rituximab kombiniert, einem monoklonalen Antikörper gegen CD 20. Auch Interferon $\alpha-2 b$ oder die autologe Stammzelltransplantation werden in der Behandlung der LYG angewendet [3].

\section{Bronchozentrische Granulomatose}

Die bronchozentrische Granulomatose (BG) ist eine nicht-angiozentrische Form der Granulomatosen und beteiligt vorwiegend die Bronchien und Bronchiolen. Sie wird daher als bronchozentrisch bezeichnet. Die Bronchien und Bronchiolen sind ektatisch, verdickt und enthalten visköses mukopurulentes oder käsiges Material [1]. Symptomatisch steht produktiver Husten mit zähem Sekret im Vordergrund.

Bronchozentrische Granulome treten bei verschiedenen Erkrankungen auf, unter anderem der ankylosierenden Spondylitis, der rheumatoiden Arthritis, der Wegener'schen Granulomatose und mykobakteriellen, fungalen oder parasitären Infektionen. Die histologischen Befunde einer BG können auch als Begleiterscheinungen eines bronchogenen Karzinoms gefunden werden [6]. Insofern setzt die Diagnose einer BG den Ausschluss einer infektiösen Ursache, einer Wegener'schen Granulomatose, eines bronchogenen Karzinoms oder anderer Erkrankungen voraus. Der histologische Nachweis von bronchozentrischen Granulomen ist somit noch nicht pathognomonisch und beweisend für eine BG. Ein Drittel bis die Hälfte der Patienten mit einer BG leiden an einem Asthma bronchiale in Kombination mit einer allergischen bronchopulmonalen Aspergillose (ABPA), die bei diesen Patienten als ursächlich für die BG angenommen wird. Es zeigen sich bei diesen Patienten histologisch Pilzhyphen und ausgeprägte eosinophile pulmonale Infiltrationen. In der bronchoalveolären Lavage (BAL) findet sich daher eine Eosinophilie. Bei den nicht-asthmatischen Patienten mit einer BG ist die Ursache unbekannt, Pilzhyphen lassen sich nicht nachweisen [7]. Eine pulmonale Arteriitis wird häufig gefunden, aber als sekundäre inflammatorische Veränderung
Tab. 2 Lymphoide Granulomatose: Schweregrade anhand histologischer Kriterien.

\begin{tabular}{|ll} 
Grad & Histologische Kriterien \\
\hline 1 & $\begin{array}{l}\text { Polymorphe mononukleäre Zell-Infiltrate mit einzelnen großen B-Zellen } \\
\text { vor dem Hintergrund reaktiver T-Zell-Infiltrate; EBV-RNA kann fehlen }\end{array}$ \\
\hline 2 & $\begin{array}{l}\text { Polymorphe mononukleäre Zell-Infiltrate mit leicht zu identifizierenden } \\
\text { großen, oft atypischen B-Zellen; EBV-RNA meist vorhanden }\end{array}$ \\
\hline 3 & $\begin{array}{l}\text { Zahlreiche B-Zellen mit nur fokal polymorphen Zell-Infiltraten; } \\
\text { EBV-RNA in vielen Zellen }\end{array}$ \\
\end{tabular}

gewertet. Extrapulmonale Manifestationen sind selten. Das Erkrankungsalter liegt hauptsächlich zwischen dem 4. und 7. Lebensjahrzehnt [8]. Die Prognose der Erkrankung ist günstig und spontane Heilungen treten auf. Insbesondere bei den asthmatischen Patienten kommt die Therapie mit einem oralen Kortikoid in Betracht.

Bei der bronchozentrischen Granulomatose wird eine nicht-asthmatische von der asthmatischen Form in Kombination mit einer bronchopulmonalen Aspergillose unterschieden.

Radiologisch können vielfältige Bilder auftreten mit multiplen oder solitären Rundherden oder Infiltrationen. Richtungweisend können Atelektasen durch eine Bronchusverlegung durch Sekret bei der ABPA sein. Eine hiläre Lymphadenopathie oder eine pleurale Beteiligung sind selten. Die radiologischen Veränderungen bilden sich unter einer oralen Kortikoidtherapie zurück.

\section{Nekrotisierende sarkoide Granulomatose}

Die seltene nekrotisierende sarkoide Granulomatose (NSG) ist charakterisiert durch das histologische Bild einer Angiitis und sarkoider Granulome mit unterschiedlicher Nekroseausprägung [1] Häufiges, aber meist einziges Symptom ist ein trockener Husten. Bei einer pleuralen Beteiligung tritt ein pleuritischer Schmerz auf. Die Erkrankung betrifft Frauen häufiger als Männer vorwiegend im jungen und mittleren Lebensalter [9]. In der radiologischen Bildgebung stellen sich bilateral pulmonale Rundherde mit einer subpleuralen oder einer peribronchovaskulären Verteilung $\operatorname{dar}(\bullet$ Abb. 2).
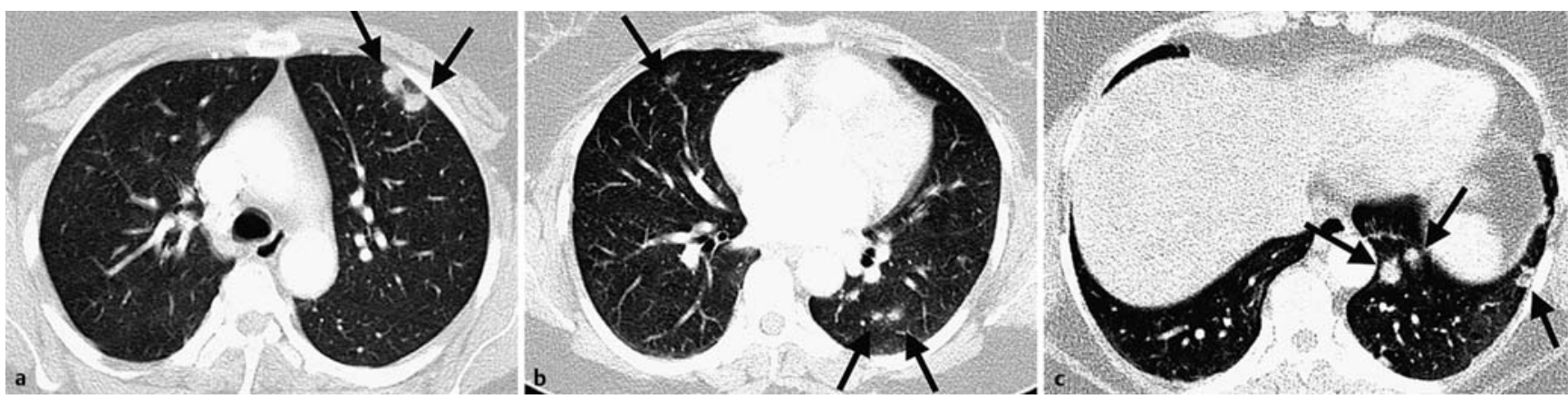

Abb. 2 Computertomografie des Thorax einer Patientin mit nekrotisierender sarkoider Ganulomatose: multiple pulmonale Rundherde (Pfeile) in allen Lungenlappen (a: oberer, b: mittlerer, c: unterer Lungenanschnitt). 
Tab. 3 Wegener'schen Granulomatose: Krankheitsstadien nach „European League Against Rheumatism“ (EULAR)/,European Vasculitis Study Group“ (EUVAS).

\begin{tabular}{|c|c|c|c|c|}
\hline $\begin{array}{l}\text { Klinische } \\
\text { Subgruppe }\end{array}$ & $\begin{array}{l}\text { Systemische } \\
\text { Vaskulitis } \\
\text { außerhalb von } \\
\text { HNO-Bereich } \\
\text { und Lunge }\end{array}$ & $\begin{array}{l}\text { Bedrohte } \\
\text { Organ- } \\
\text { funktion }\end{array}$ & $\begin{array}{l}\text { Weitere } \\
\text { Kriterien }\end{array}$ & $\begin{array}{l}\text { Serum- } \\
\text { kreatinin } \\
{[\mu \mathrm{mol} / \mathrm{l}]}\end{array}$ \\
\hline lokalisiert & nein & nein & $\begin{array}{l}\text { keine B-Symp- } \\
\text { tome, ANCA } \\
\text { meist negativ }\end{array}$ & $<120$ \\
\hline frühsystemisch & ja & nein & $\begin{array}{l}\text { B-Symptome, } \\
\text { ANCA positiv } \\
\text { oder negativ }\end{array}$ & $<120$ \\
\hline generalisiert & ja & ja & ANCA positiv & $<500$ \\
\hline schwer & ja & $\begin{array}{l}\text { Organ- } \\
\text { versagen }\end{array}$ & ANCA positiv & $>500$ \\
\hline refraktär & ja & ja & $\begin{array}{l}\text { refraktär auf } \\
\text { eine Standard- } \\
\text { therapie }\end{array}$ & jeder Wert \\
\hline
\end{tabular}

Auch ein Mischbild aus multiplen Rundherden und diffusen Infiltraten kann auftreten. Bei einer pleuralen Beteiligung zeigt sich eine diffuse pleurale Verdickung. Im Gegensatz zur Sarkoidose, bei der meist die mediastinalen oder hilären Lymphknoten mitbetroffen sind, ist bei der NSG ein Befall mediastinaler und hilärer Lymphknoten selten. Die NSG kann spontan oder unter Therapie mit einem oralen Kortikoid eine Remission zeigen und hat einen meist benignen Verlauf [10].

Die nekrotisierende sarkoide Granulomatose hat einen günstigen klinischen Verlauf unter der Behandlung mit einem oralen Kortikoid oder auch spontan.

\section{Wegener'sche Granulomatose}

Die Wegener'sche Granulomatose (WG) führt zu einer nekrotisierenden Vaskulitis mit ulzerierenden, nicht verkäsenden Granulomen und kann den gesamten Respirationstrakt betreffen. Ulzerationen finden sich im Oropharynx, und eine chronische Rhinitis, Sinusitis oder Otitis media können vorhanden sein. Neben Schmerzen in den Kieferhöhlen und Ausfluss von eitrigem oder blutigem Sekret kann eine seröse Otitis media zur Verlegung der Tuba auditiva Eustachii führen und Hörstörungen verursachen. Husten und Hämoptysen treten als Folge der pulmonalen Beteiligung auf. Stenosierungen der Atemwege können insbesondere subglottisch oder tracheal zu ausgeprägter Dyspnoe und zu einem inspiratorischen Stridor führen. Das Bild der Hautmanifestationen ist vielfältig: Ulzera, subkutane Knötchen, Bläschen, palpable Purpura, Papeln. Eine Augenbeteiligung ist möglich und in ihrer Ausprägung sehr variabel. Unterschieden wird ein lokal begrenztes Stadium, bei dem ausschließlich der Respirationstrakt betroffen ist, von einem generalisierten vaskulitischen Stadium, bei dem zusätzlich meist eine Glomerulonephritis vorliegt. Im generalisierten Stadium können weitere Symptome durch eine vas- kulitische Beteiligung entstehen, z.B. der Augen, der Muskulatur oder des zentralen Nervensystems. Eine Beteiligung des Tracheobronchialbaums tritt in 59\% der Fälle auf und kann zu subglottischen, trachealen oder bronchialen Stenosierungen und zu einer ulcerierenden Tracheobronchitis führen [11]. Vorhandene Organschäden wirken sich ungünstig auf die Prognose der WG aus. Etwa 5\% der Patienten verbleiben im lokalisierten Stadium und entwickeln keine systemische Vaskulitis [12]. Von der „European Vasculitis Study Group“ (EUVAS) und der „Wegener's Granulomatosis Etanercept Trial“ (WGET) Studiengruppe wurden Kriterien für die Beurteilung der Erkrankungsschwere definiert. Diese wurden ursprünglich für die Beurteilung der Erkrankung im Rahmen von Studien entwickelt, finden aber im klinischen Alltag Anwendung zur Therapiewahl und zur Einschätzung der Prognose. Eine einheitliche Empfehlung ist in Bearbeitung. Bis dahin werden beide Beurteilungen von der „European League Against Rheumatism“ (EULAR) empfohlen [13]. Tab. 3 zeigt die Krankheitsstadien nach EULAR/EUVAS-Definition.

Antineutrophile zytoplasmatische Antikörper mit zytoplasmatischem Fluoreszenzmuster (cANCA) können bei $67 \%$ der Patienten mit aktiver Erkrankung im lokalisierten Stadium und bei $96 \%$ der Patienten im generalisierten Stadium mit einer Spezifität von 99\% nachgewiesen werden [14]. Zielantigen der cANCA ist häufig die Proteinase 3 (PR3-ANCA). In der BAL tritt eine Neutrophilie auf. Radiologisch zeigen sich bilateral multiple pulmonale Rundherde mit Durchmessern von $5 \mathrm{~mm}$ bis $10 \mathrm{~cm}$. Die Läsionen sind typischerweise scharf begrenzt, ohne einen Lungenbereich zu bevorzugen, wobei die Lungenspitzen ausgespart zu sein scheinen. Die Rundherde sind zum Teil eingeschmolzen und werden dann als Pseudokavernen bezeichnet ( $\bullet$ Abb. 3). Die Anzahl und Größe der pulmonalen Herde korreliert zur Krankheitsaktivität [15].

\section{Therapie der WG}

Die Therapie richtet sich nach dem Stadium der Erkrankung. Im lokalisierten Stadium kann Cotrimoxazol zu einer längerfristigen Remission führen. Bei fehlendem Therapieansprechen wird die Therapie des lokalisierten Stadiums auf Medikamente zur Erhaltungstherapie des generalisierten Stadiums eskaliert. Bei frühsystemischem, leichterem Erkrankungsbild der WG ohne lebensbedrohliche Organmanifestationen ist Methotrexat oder Azathioprin kombiniert mit Prednisolon geeignet. Im generalisierten Stadium mit organbedrohlichem Verlauf wird zunächst eine Remissionsinduktion angestrebt. Als Standardtherapie wird hierzu eine Kombinationstherapie aus Prednisolon und Cylophosphamid angewendet. Das Cyclophosphamid kann täglich oral über drei bis sechs Monate oder in Anlehnung an das Protokoll 

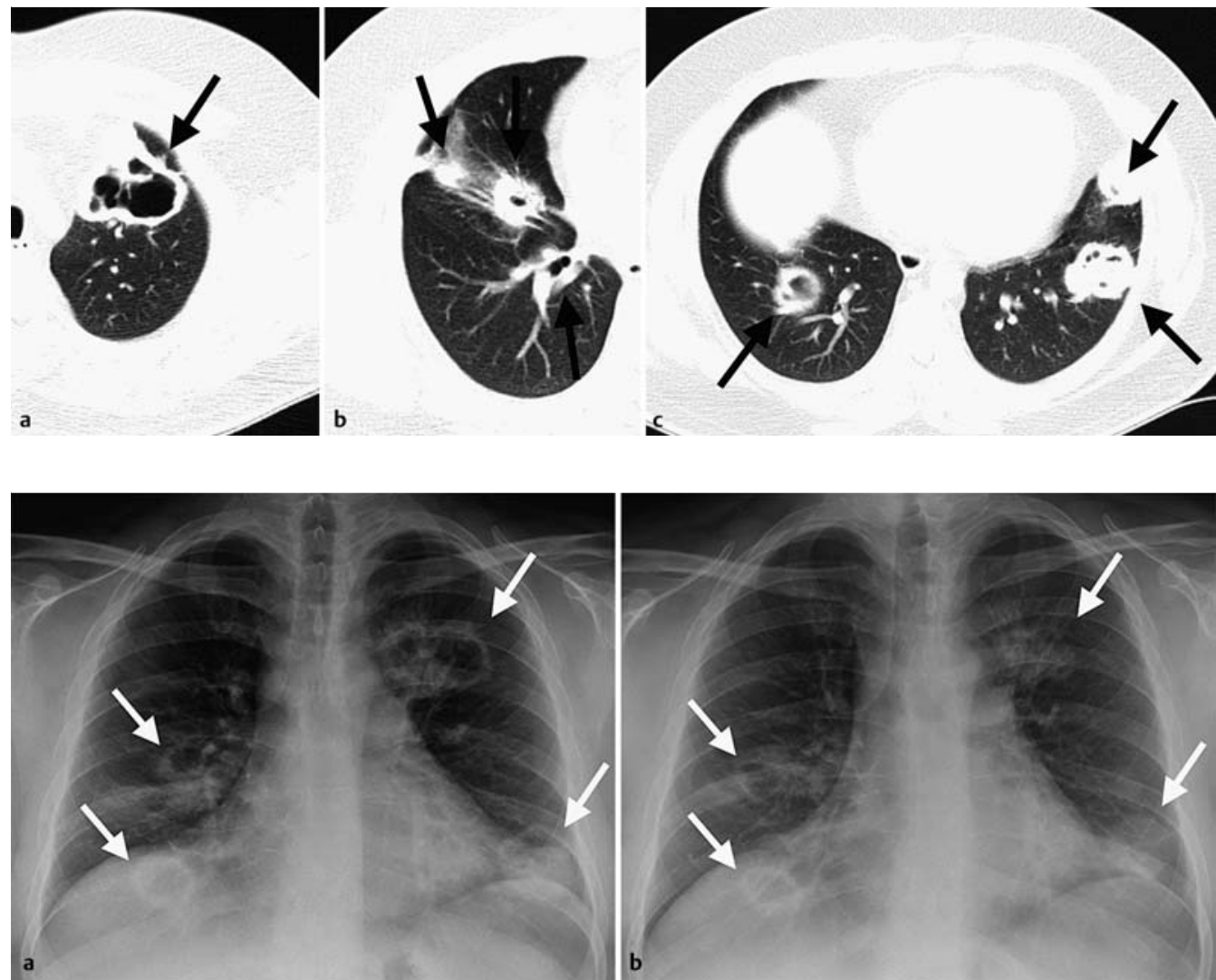

der CYCLOPS-Studie sechsmal intravenös als Bolus im Abstand von zwei bis drei Wochen erfolgen [16]. Mit zunehmender Kumulativdosis des Cyclophosphamids steigt das Risiko eines Urothelkarzinoms und durch die Knochenmarkschädigung das Risiko einer akuten myeloischen Leukämie und eines sekundären myelodysplastischen Syndroms. Um das Risiko eines Blasenkarzinoms und einer hämorrhagischen Zystistis unter der Therapie mit Cyclophosphamid zu reduzieren, ist eine ausreichend hohe Trinkmenge des Patienten zu beachten und die Gabe von Uromitexan unter einer Stoßtherapie indiziert. Unter einer adäquaten Induktionstherapie erreichen rund $90 \%$ der Patienten eine Remission ( $\bullet$ Abb. 4).

Bei fehlendem Therapieansprechen ist in lebensbedrohlichen Fällen eine Plasmapherese zu erwägen. Als weitere Optionen zur Remissionsinduktion kommen Infliximab, Rituximab, Immunglobuline, Mycophenolatmofetil, 15-Deoxyspergualin oder Anti-Thymozytenglobulin in Betracht [17]. Zur Erhaltungstherapie wird Methotrexat oder Azathioprin kombiniert mit Prednisolon angewendet. Prednisolon sollte sechs Monate nach Therapiebeginn auf eine Tagesdosis von 5-10 mg reduziert sein. Für die Dauer der Erhaltungstherapie liegen keine gesicherten Empfehlungen vor, allgemein empfohlen werden 18-24 Monate.
Das therapeutische Vorgehen bei der Wegener'schen Granulomatose ist abhängig vom Vorliegen lebens- oder organbedrohlicher Manifestationen. In diesem Fall erfolgt zunächst eine Induktionstherapie, gefolgt von einer Erhaltungstherapie.

\section{Churg-Strauss-Syndrom}

Bei Patienten mit einem Churg-Strauss-Syndrom (CSS) stehen häufig asthmatische Beschwerden im Vordergrund. Als Ausdruck der Systemerkrankung können Fieber, Appetitlosigkeit und allgemeine körperliche Schwäche auftreten. Monooder Polyneuropathien treten auf und ein vaskulitischer cerebraler Befall ist möglich. Bei Beteiligung der Haut besteht eine Purpura mit kutaner und subkutaner Knotenbildung. Histologisch findet sich eine granulomatöse, nekrotisierende Vaskulitis. Sie betrifft vorwiegend die kleinen Gefäße und geht mit eosinophilen Infiltraten einher. Die BAL zeigt in der Regel eine Eosinophilie. Die Inzidenz von Thromboembolien ist erhöht. Im Gegensatz zur Wegener'schen Granulomatose ist eine Nierenbeteiligung selten. Die häufigste Todesursache beim CSS sind bei einer kardialen Beteilung der Myokardinfarkt durch eine Vaskulitis der Koronarien und die Herzinsuffizienz durch eine eosinophile granulomatöse Myokarditis. Häufig entwickelt sich das CSS über drei ineinander übergehende Stadien. Nach einem Prodromalstadium mit Asthma bronchiale und allergischen Manifestationen folgt eine eosinophile Infiltration vor allem des Lungengewebes und des
Abb. 3 Computertomografie des Thorax eines 35-jährigen Patienten mit progredientem pulmonalem Befall (Pfeile) einer Wegener'schen Granulomatose trotz Therapie mit Azathioprin und Prednisolon (a: Lungenoberlappen links, b: Mittellappen, c: Unterlappen beidseits).

Abb. 4 Röntgen des Thorax des gleichen Patienten wie in - Abb. 3 mit Regression der Manifestationen (Pfeile) unter einer Induktionstherapie mit Cyclophosphamid und Prednisolon (a: vor, b: nach 6 Zyklen).

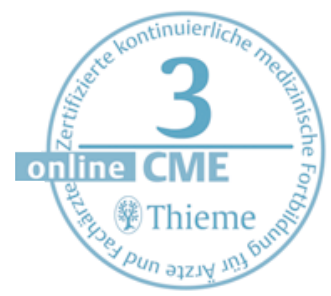


Abb. 5 Computertomografie des Thorax einer 20-jährigen Patientin mit einem ChurgStrauss-Syndrom: baumwollartige, in die Peripherie ausstrahlende Verdichtungen in den Oberlappen beidseits.

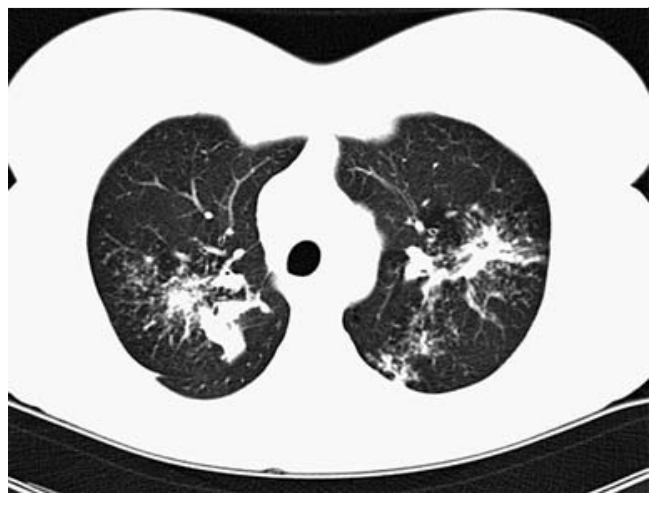

Tab. 4 Wegener'sche Granulomatose und Churg-Strauss-Syndrom: Krankheitskriterien der „American College of Rheumatology“ (ACR) und Definitionen der „Chapel Hill Consensus Conference" ( $\mathrm{CHCC})$.

\begin{tabular}{|c|c|c|}
\hline & ACR-Kriterien & Definition CHCC \\
\hline $\begin{array}{l}\text { Wegener'sche } \\
\text { Granulomatose }\end{array}$ & $\begin{array}{l}\text { mindestens } 2 \text { von } 4 \text { Kriterien: } \\
\text { - nasale oder orale Entzündung } \\
\text { - Kavernen, Infiltrate oder } \\
\text { Granulome im Röntgen des } \\
\text { Thorax } \\
\text { - pathologisches Urinsediment } \\
\text { mit Mikrohämaturie oder } \\
\text { Erythrozytenzylindern } \\
\text { - histologisch granulomatöse } \\
\text { Entzündung in arteriellen } \\
\text { Gefäßwänden oder peri- } \\
\text { vaskulär }\end{array}$ & $\begin{array}{l}\text { - granulomatöse Entzündung } \\
\text { des Respirationstraktes } \\
\text { - nekrotisierende Entzündung } \\
\text { kleiner bis mittelgroßer } \\
\text { Gefäße } \\
\text { - häufig mit nekrotisierender } \\
\text { Glomerulonephritis }\end{array}$ \\
\hline $\begin{array}{l}\text { Churg-Strauss- } \\
\text { Syndrom }\end{array}$ & $\begin{array}{l}\text { mindestens } 4 \text { von } 6 \text { Kriterien: } \\
\text { - Asthma bronchiale } \\
\text { - pulmonale Infiltrate } \\
\text { - Eosinophilie }>10 \% \\
\text { - Mono- oder Polyneuropathie } \\
\text { - Sinusitis } \\
\text { - histologisch extravaskuläre } \\
\text { Eosinophilie }\end{array}$ & $\begin{array}{l}\text { - eosinophilenreiche und } \\
\text { granulomatöse Entzündung } \\
\text { des Respirationstraktes } \\
\text { - nekrotisierende Entzündung } \\
\text { kleiner bis mittelgroßer } \\
\text { Gefäße } \\
\text { - assoziiert mit Asthma und } \\
\text { Eosinophilie }\end{array}$ \\
\hline
\end{tabular}

Myokards. Im dritten, systemischen Stadium entwickelt sich eine nekrotisierende Vaskulitis mit Beteiligung der Haut, der peripheren Nerven und der Nieren [18]. Montelukast und Omalizumab werden als Trigger für eine Progression des CSS diskutiert. Beim Progressionsrisiko durch Montelukast kann nicht ausgeschlossen werden, dass eine generelle Eskalation der Asthmatherapie bei einer schwereren Verlaufsform des Asthmas ursächlich ist, denn auch langwirksame inhalative $\beta_{2}$-Sympathomimetika und orale Kortikoide erhöhen statistisch das Risiko [19]. Nicht Omalizumab selbst, sondern der kortikoidsparende Effekt des Medikaments könnte womöglich die Progression des CSS begünstigen [20].

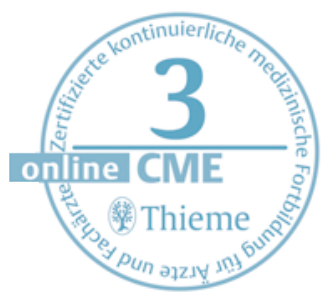

Antineutrophile zytoplasmatische Antikörper, mit perinukleärem Fluoreszenzmuster (pANCA) gegen die Myeloperoxidase gerichtet (MPO-ANCA), lassen sich bei $40 \%$ der Patienten nachweisen. Im Blutbild sowie in den befallenen Organen zeigt sich eine Eosinophilie. Es kann zwischen einem pANCA-assoziierten vaskulitischen Verlauf und einem pANCA-negativen Verlauf unterschieden werden, der mit einer eosinophilen Organmanifestation einhergeht; Patienten mit kardialer Beteiligung sind meist ANCA-negativ, zeigen aber eine ausgeprägtere Eosinophilie [18]. Eine sensitive kardiale Diagnostik, mit der auch subklinische kardiale Manifestationen des CSS gesichert werden können, ist mit der kardialen Magnetresonanztomografie möglich.

Wesentliches Charakteristikum des Churg-Strauss-Syndroms ist eine Bluteosinophilie und Eosinophilie der befallenen Organe.

Radiologisch kann ein Normalbefund bestehen. Im Prodromalstadium zeigen sich häufig flüchtige pulmonale Infiltrate. Im Stadium eosinophiler Infiltrationen können symmetrische perihiläre oder periphere pulmonale Verdichtungen auftreten ( $\bullet$ Abb.5). Im vaskulitischen Stadium sind die pulmonalen Verdichtungen meist großflächig und peripher, oder es zeigen sich diffus miliare Rundherde [21].

\section{Therapie CSS}

Erstmalig 1996 wurde auf der Grundlage einer Datenbank der „French Vasculitis Study Group“ für die systemischen nekrotisierenden Vaskulitiden Panarteriitis nodosa, mikroskopische Polyangiitis und das Churg-Strauss-Syndorm ein Score mit fünf Punkten zur Beurteilung der Krankheitsprognose anhand der Mortalität zum Zeitpunkt der Diagnosestellung entwickelt [22]. Proteinurie $>1 \mathrm{~g} /$ Tag, Serumkreatinin $>140 \mu \mathrm{mol} / \mathrm{l}$, krankheitsspezifische Kardiomyopathie, gastrointestinale Beteiligung und Beteiligung des zentralen Nervensystems gehen mit einem erhöhten Mortalitätsrisiko einher. Wenn keines der Kriterien vorliegt, ist von einer guten Prognose auszugehen, und die Therapie mit einem oralen Kortikoid führt in $90 \%$ der Fälle zu einer Remission. Nach einem mittleren Verlauf von 56 Monaten besteht eine Rezidivrate von 35\%, und bei mehr als 75\% der Patientin kann aufgrund des schweren Asthma bronchiale das orale Kortikoid nicht vollständig ausgeschlichen werden [23]. Beim Vorliegen mindestens eines Risikomerkmals sollte zunächst in Anlehnung an die Wegener'sche Granulomatose eine Induktionstherapie mit einer Kombination von Cyclophosphamid und einem oralen Kortikoid zur Remission erfolgen. Anschließend kann eine Erhaltungstherapie mit einer Kombination von oralem Kortikoid und wahlweise Azathioprin oder Methotrexat erfolgen. Die Dosis des Kortikoids wird im Verlauf soweit möglich reduziert. Die Dauer einer optimalen Erhaltungstherapie ist unklar. Für die Gesamtdauer der Induktions- und Erhaltungstherapie werden mindestens 18-24 Monate empfohlen. Die Behandlung des CCS mit Rituximab, dem monoklonalen Antikörper gegen CD 20, ist bislang noch umstritten. Für therapierefraktäre Erkrankungen kommen Interferon- $\alpha$, der Tyrosinkinaseinhibitor Imatinib, Antikörper 
Tab. 5 Differenzialdiagnose der verschiedenen Formen pulmonaler Angiitis und Granulomatosen.

\begin{tabular}{|c|c|c|c|c|c|c|}
\hline & Symptome & Radiologie & Histologie & $\begin{array}{l}\text { typischer } \\
\text { Befall }\end{array}$ & Labor & $\begin{array}{l}\text { Besonder- } \\
\text { heiten }\end{array}$ \\
\hline $\begin{array}{l}\text { Lymphomatoide } \\
\text { Granulomatose }\end{array}$ & $\begin{array}{l}\text { Husten, } \\
\text { B-Symptomatik }\end{array}$ & $\begin{array}{l}\text { RH vorwiegend in } \\
\text { Ober- und Mittel- } \\
\text { feldern bilateral, } \\
\text { auch einseitig; } \\
\text { Einschmelzungen; } \\
\text { retikulonoduläre } \\
\text { Zeichnungsver- } \\
\text { mehrung }\end{array}$ & $\begin{array}{l}\text { granulomatöse } \\
\text { Veränderungen mit } \\
\text { zentraler Nekrose, } \\
\text { knotige lymphoide } \\
\text { Infiltrate, Angiitis } \\
\text { mit transmuraler } \\
\text { lymphozytärer } \\
\text { Infiltration }\end{array}$ & $\begin{array}{l}\text { Lunge, Haut, } \\
\text { peripheres } \\
\text { und zentrales } \\
\text { Nervensys- } \\
\text { tem }\end{array}$ & $\begin{array}{l}\text { kein spezifischer } \\
\text { Marker }\end{array}$ & $\begin{array}{l}\text { assoziiert mit } \\
\text { dem Ebstein- } \\
\text { Barr-Virus }\end{array}$ \\
\hline $\begin{array}{l}\text { Bronchozentrische } \\
\text { Granulomatose }\end{array}$ & $\begin{array}{l}\text { produktiver Hus- } \\
\text { ten mit zähem } \\
\text { Sekret; bei ABPA: } \\
\text { Asthma bronchiale }\end{array}$ & $\begin{array}{l}\text { vielfältig; multiple } \\
\text { oder solitäre RH oder } \\
\text { Infiltrate, Bronchi- } \\
\text { ektasen; bei ABPA } \\
\text { Atelektasen möglich }\end{array}$ & $\begin{array}{l}\text { bronchozentrische } \\
\text { Granulome; ekta- } \\
\text { tische, verdickte } \\
\text { Bronchien und Bron- } \\
\text { chiolen mit viskösem } \\
\text { Material; bei ABPA } \\
\text { eosinophile Infil- } \\
\text { trate, Pilzhyphen; } \\
\text { sekundäre pulmo- } \\
\text { nale Arteriitis }\end{array}$ & Lunge & $\begin{array}{l}\text { bei ABPA: Blut- } \\
\text { eosinophilie, } \\
\text { IgE und IgG Anti- } \\
\text { körper gegen } \\
\text { Aspergillen, } \\
\text { Eosinophilie in } \\
\text { BAL }\end{array}$ & $\begin{array}{l}\text { asthmatische } \\
\text { und nicht- } \\
\text { asthmatische } \\
\text { Form }\end{array}$ \\
\hline $\begin{array}{l}\text { Nekrotisierende } \\
\text { sarkoide Granulo- } \\
\text { matose }\end{array}$ & $\begin{array}{l}\text { trockener } \\
\text { Husten }\end{array}$ & $\begin{array}{l}\text { subpleurale oder } \\
\text { peribronchiale RH } \\
\text { beidseits; auch } \\
\text { Mischbild aus mul- } \\
\text { tiplen RH und dif- } \\
\text { fusen Infiltraten; } \\
\text { diffuse pleurale } \\
\text { Verdickung; media- } \\
\text { stinale und hiläre } \\
\text { Lymphknoten meist } \\
\text { unauffällig }\end{array}$ & $\begin{array}{l}\text { sarkoide Granulome } \\
\text { mit unterschiedli- } \\
\text { cher Nekroseausprä- } \\
\text { gung, Angiitis }\end{array}$ & Lunge & $\begin{array}{l}\text { kein spezifischer } \\
\text { Marker }\end{array}$ & \\
\hline $\begin{array}{l}\text { Wegener'sche } \\
\text { Granulomatose }\end{array}$ & $\begin{array}{l}\text { Rhinitis/Sinusitis } \\
\text { mit eitrigem oder } \\
\text { blutigen Sekret, } \\
\text { Ulzerationen im } \\
\text { Oropharynx; } \\
\text { Hämoptysen; } \\
\text { bei systemischer } \\
\text { Vaskulitis: B-Symp- } \\
\text { tomatik, Arthral- } \\
\text { gien, Myalgien, } \\
\text { periphere Neuro- } \\
\text { pathien, ZNS- } \\
\text { Symptome }\end{array}$ & $\begin{array}{l}\text { RH bilateral, } \\
\text { Pseudokavernen }\end{array}$ & $\begin{array}{l}\text { nekrotisierende } \\
\text { Vaskulitis mit intra- } \\
\text { und extravaskulären } \\
\text { Granulomen; Niere: } \\
\text { zunächst segmen- } \\
\text { tale Glomerulitis, } \\
\text { dann progressive } \\
\text { Glomerulonephritis }\end{array}$ & $\begin{array}{l}\text { Respirations- } \\
\text { trakt, Nieren, } \\
\text { Haut, Augen }\end{array}$ & $\begin{array}{l}\text { CANCA mit Ziel- } \\
\text { antigen PR3 } \\
\text { (PR3-ANCA); } \\
\text { Anstieg des } \\
\text { Serumkreati- } \\
\text { nins, Erythrozy- } \\
\text { turie bei Glome- } \\
\text { rulonephritis; } \\
\text { Neutrophilie und } \\
\text { alveoläre Hämor- } \\
\text { rhagie in BAL }\end{array}$ & $\begin{array}{l}\text { tracheale } \\
\text { und bron- } \\
\text { chiale Steno- } \\
\text { sen; Perfora- } \\
\text { tion des } \\
\text { Nasensep- } \\
\text { tums mit } \\
\text { Folge einer } \\
\text { Sattelnase }\end{array}$ \\
\hline $\begin{array}{l}\text { Churg-Strauss- } \\
\text { Syndrom }\end{array}$ & $\begin{array}{l}\text { Asthma bron- } \\
\text { chiale, Rhinitis/ } \\
\text { Sinusitis, Häm- } \\
\text { optysen, Mono-/ } \\
\text { Polyneuropathie, } \\
\text { B-Symptomatik }\end{array}$ & $\begin{array}{l}\text { im Prodromal- } \\
\text { stadium flüchtige } \\
\text { Infiltrate; symmetri- } \\
\text { sche perihiläre oder } \\
\text { periphere Verdich- } \\
\text { tungen oder diffuse } \\
\text { miliare RH; Normal- } \\
\text { befund möglich }\end{array}$ & $\begin{array}{l}\text { granulomatöse } \\
\text { Veränderungen des } \\
\text { Gewebes und vor- } \\
\text { wiegend der kleinen } \\
\text { Gefäße, assoziiert } \\
\text { mit eosinophiler } \\
\text { Gewebeinfiltration }\end{array}$ & $\begin{array}{l}\text { Lunge, Herz, } \\
\text { Haut, peri- } \\
\text { pheres Ner- } \\
\text { vensystem, } \\
\text { Gastrointes- } \\
\text { tinaltrakt }\end{array}$ & $\begin{array}{l}\text { pANCA gegen } \\
\text { Myeloperoxidase } \\
\text { ( } 40 \% \text { d. F.), } \\
\text { Eosinophilie } \\
>10 \% \text { im Diffe- } \\
\text { renzialblutbild; } \\
\text { Eosinophilie und } \\
\text { alveoläre Hämor- } \\
\text { rhagie in BAL }\end{array}$ & $\begin{array}{l}\text { gehäuft } \\
\text { Thrombo- } \\
\text { embolien; } \\
\text { bei kardialer } \\
\text { Beteiligung } \\
\text { hohe Letali- } \\
\text { tät }\end{array}$ \\
\hline
\end{tabular}

Abkürzungen: B-Symptomatik: Fieber, Nachtschweiß, Gewichtsverlust; RH: Rundherde; ABPA: allergische bronchopulmonale Aspergillose

gegen IL-5 und monoklonale Antikörper gegen den Tumornekrosefaktor $\alpha$ (Infliximab und Etanercept) in Betracht [18].

Wegener'sche Granulomatose und ChurgStrauss-Syndrom: Differenzialdiagnose und gemeinsamer Risikoscore

Von dem „American College of Rheumatology“ (ACR) wurden 1990 Kriterien [24] und von der „Chapel Hill Consensus Conference“ (CHCC) 1992 Definitionen [25] für die Einordnung einer gesi- cherten Vaskulitis als WG oder als CSS verfasst. Sie finden im klinischen Alltag Anwendung zur Erhärtung einer Verdachtsdiagnose. Gegenüberstellend sind die Kriterien und Definitionen in - Tab. 4 aufgeführt.

Beide Erkrankungen können in der BAL eine alveoläre Hämorrhagie zeigen. Sie ist bei der WG häufig, beim CSS selten und prädisponiert für bakterielle Superinfektionen. Differenzialdiagnostisch kann eine diffuse alveoläre Hämorrhagie idiopathisch, beim Goodpasture-Syndrom, einer

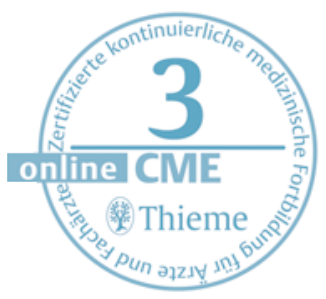


Kollagenose oder einer Immunkomplexerkrankung auftreten.

Bei der Wegener'schen Granulomatose und dem ChurgStrauss-Syndrom kann die BAL eine Hämorrhagie zeigen.

Der für die Therapiewahl des CSS verwendete 5-Punkte-Score wurde kürzlich überarbeitet und gilt nun auch für die WG. Risikofaktoren sind: Alter > 65 Jahre, erkrankungsbedingte kardiale Symptome, gastrointestinale Beteiligung und Niereninsuffizienz (Kreatinin $\geq 150 \mu \mathrm{mol} / \mathrm{L}$ ). Sie werden jeweils mit einem Punkt bewertet. Eine Beteiligung von Hals, Nase und Ohren korreliert mit einem verminderten Mortalitätsrisiko, sodass ihr Fehlen auch mit einem Punkt bewertet wird. Die 5-Jahres-Mortalitätsrate für die Punktsummen 0, 1 und $\geq 2$ waren $9 \%, 21 \%$ und $40 \%$ [22].

Bei der Wegner'schen Granulomatose und dem ChurgStrauss-Syndrom erhöhen folgende Faktoren das Mortalitätsrisiko: Alter > 65 Jahre, krankheitsbedingte kardiale Symptome, gastrointestinale Beteiligung, Niereninsuffizienz, Fehlen einer Hals-, Nasen- oder Ohren-Beteiligung.

\section{Zusammenfassung}

Ursächlich für multiple pulmonale Rundherde kann differenzialdiagnostisch eine Gruppe von Erkrankungen sein, die histologisch eine pulmonale Angiitis und Granulomatose zeigen. Hierbei handelt es sich durchweg um seltene Erkrankungen, die einer speziellen differenzialdiagnostischen Abklärung bedürfen.

Pulmonale Angiitis und Granulomatose treten bei der lymphomatoiden Granulomatose, der bronchozentrischen Granulomatose, der nekrotisierenden sarkoiden Granulomatose, der Wegener'schen Granulomatose und dem ChurgStrauss-Syndrom auf. Differenzialdiagnostische Charakteristika sind in $\bullet$ Tab. 5 gegenübergestellt.

Die BAL kann differenzialdiagnostische Hinweise liefern. Eine alveoläre Hämorrhagie tritt beim CSS und der WG auf. Die Differenzialzytologie der BAL zeigt bei der WG eine Neutrophilie und beim CSS und der ABPA eine Eosinophilie. Bei zweifelhaften zytologischen oder histologischen Befunden der bronchoskopisch gewonnenen Proben ist eine chirurgische Lungenbiopsie zur Diagnosesicherung indiziert. Die histologische Verteilung der Granulome ist hinweisend auf die zugrunde liegende Erkrankung. Die definitive Diagnose kann aber erst in Zusammenschau von Klinik, radiologischen Befunden, Autoantikörperdiagnostik und Histologie nach Ausschluss infektöser und maligner Differentialdiagnosen gestellt werden.
Die Prognose und die Therapie der verschiedenen Erkrankungen sind trotz der histologischen Gemeinsamkeiten sehr unterschiedlich. Ein extrapulmonaler Organbefall geht mit einer schwereren Erkrankung und schlechteren Prognose einher.

\section{Interessenkonflikt}

$\nabla$

Die Autoren geben an, dass kein Interessenkonflikt besteht.

\section{Literatur}

1 Liebow AA. The J. Burns Amberson lecture - pulmonary angiitis and granulomatosis. Am Rev Respir Dis 1973; 108: 1 - 18

2 Bräunlich J, Seyfarth HJ, Gessner C et al. Lymphomatoide Granulomatose - eine kurze Darstellung der Erkrankung anhand eines außergewöhnlichen Falles. Pneumologie 2009; 63: 697 - 701

3 Katzenstein AL, Doxtader E, Narendra S. Lymphomatoid granulomatosis: insights gained over 4 decades. Am J Surg Pathol 2010; 34: 35-48

4 Dee PM, Arora NS, Innes DJ Jr. The pulmonary manifestations of lymphomatoid granulomatosis Radiology 1982; 143: $613-618$

5 Fauci AS, Haynes BF, Costa J et al. Lymphomatoid Granulomatosis. Prospective clinical and therapeutic experience over 10 years. N Engl J Med 1982; 306: 68-74

6 Houser SL, Mark EJ. Bronchocentric granulomatosis with mucus impaction due to bronchogenic carcinoma. An association with clinical relevance. Arch Pathol Lab Med 2000; 124: $1168-1171$

7 Myers JL, Katzenstein AL. Granulomatous infection mimicking bronchocentric granulomatosis. Am J Surg Pathol 1986; 10: 317-22

8 Koss MN, Robinson RG, Hochholzer L. Bronchocentric granulomatosis. Hum Pathol 1981; 12: 632 - 638

9 Chittock DR, Joseph MG, Paterson NA et al. Necrotizing sarcoid granulomatosis with pleural involvement. Clinical and radiographic features. Chest 1994; 106: 672-676

10 Frechen D, Cornelissen C, Schreiner K, Jäkel J, Krüger S. Nekrotisierende sarkoidale Granulomatose der Lunge bei einer Patientin mit primärer biliärer Leberzirrhose. Dtsch Med Wochenschr 2010; 135: 1733-1736

11 Daum TE, Specks U, Colby TV et al. Tracheobronchial involvement in Wegener's granulomatosis. Am J Respir Crit Care Med 1995; 151: 522-526

12 Holle JU, Gross WL, Holl-Ulrich K et al. Prospective longterm follow-up of patients with localised Wegener's granulomatosis: does it occur as persistent disease stage? Ann Rheum Dis 2010; 69: 1934-1939

13 Hellmich B, Flossmann O, Gross WL et al. EULAR recommendations for conducting clinical studies and/or clinical trials in systemic vasculitis: focus on anti-neutrophil cytoplasm antibody-associated vasculitis. Ann Rheum Dis 2007; 66: 605-617

14 Nölle B, Specks U, Lüdemann J et al. Anticytoplasmic autoantibodies: their immunodiagnostic value in Wegener granulomatosis. Ann Intern Med 1989; 111: 28 - 40

15 Cordier JF, Valeyre D, Guillevin L et al. Pulmonary Wegener's granulomatosis. A clinical and imaging study of 77 cases. Chest 1990; 97: $906-912$

16 de Groot K, Harper L, Jayne DR et al. EUVAS (European Vasculitis Study Group). Pulse versus daily oral cyclophosphamide for induction of remission in antineutrophil cytoplasmic antibody-associated vasculitis: a randomized trial. Ann Intern Med 2009; 150: 670-680 
17 Wiesner O, Haubitz M. Granulomatöse ANCA-assoziierte Vaskulitiden. Wegener-Granulomatose. Pneumologie 2008; 5: 308 - 319

18 Pagnoux C, Guillevin L. Churg-Strauss syndrome: evidence for disease subtypes? Curr Opin Rheumatol 2010; 22: $21-28$

19 Hauser T, Mahr A, Metzler C et al. The leucotriene receptor antagonist montelukast and the risk of Churg-Strauss syndrome: a case-crossover study. Thorax 2008; 63: $677-682$

20 Wechsler ME, Wong DA, Miller MK et al. Churg-strauss syndrome in patients treated with omalizumab. Chest 2009; 136: 507 - 518

21 Frazier AA, Rosado-de-Christenson ML, Galvin JR et al. Pulmonary angiitis and granulomatosis: radiologic-pathologic correlation. Radiographics 1998; 18: 687-710
22 Guillevin L, Pagnoux C, Seror R et al. French Vasculitis Study Group. The Five-Factor Score revisited: assessment of prognoses of systemic necrotizing vasculitides based on the French Vasculitis Study Group (FVSG) cohort. Medicine (Baltimore) 2011; 90: 19-27

23 Ribi C, Cohen P, Pagnoux C et al. French Vasculitis Study Group. Treatment of Churg-Strauss syndrome without poor-prognosis factors: a multicenter, prospective, randomized, open-label study of seventy-two patients. Arthritis Rheum 2008; 58: 586 - 594

24 Fries JF, Hunder GG, Bloch DA et al. The American College of Rheumatology 1990 criteria for the classification of vasculitis. Summary. Arthritis Rheum 1990; 33: 11351136

25 Jennette JC, Falk RJ, Andrassy K et al. Nomenclature of systemic vasculitides. Proposal of an international consensus conference. Arthritis Rheum 1994; 37: 187-192 


\section{CME-Fragen Differenzialdiagnose und -thera- pie pulmonaler Erkrankungen mit Angiitis und Granulomatose}

1 Welche Aussage ist richtig?

Die lymphomatoide Granulomatose

A ist assoziiert mit dem Zytomegalievirus.

B hat eine günstige Prognose.

C ist eine lymphoproliferative Erkrankung.

D entspricht im Schweregrad 3 einem T-Zell-Lymphom.

E tritt meist im siebten Lebensjahrzehnt auf.

2 Welche Aussage ist richtig?

Die nekrotisierende sarkoide Granulomatose

A muss mit einer oralen Kortikoidtherapie behandelt werden.

B betrifft Männer häufiger als Frauen.

C manifestiert sich häufig in den mediastinalen Lymphknoten.

D zeigt histologisch sarkoide Granulome mit unterschiedlicher Nekroseausprägung.

E wird mit Cyclophosphamid als Induktionstherapie behandelt.

3 Welche Aussage ist falsch?

Die Remissionsinduktion einer Wegener'schen Granulomatose

A ist im generalisierten Stadium mit lebens- oder organbedrohlichen Manifestationen indiziert.

B erfolgt mindestens über 12 Monate.

C kann durch die Behandlung mit Cyclophosphamid zu Urothelkarzinomen führen.

D wird von einer Erhaltungstherapie gefolgt.

E erfolgt in therapierefraktären lebensbedrohlichen Fällen mittels Plasmapherese.

4 Welche Aussage ist richtig?

Antineutrophile zytoplasmatische Antikörper (ANCA) bei der Wegener'schen Granulomatose

A haben ein perinukläres Fluoreszenzmuster.

B treten häufiger bei der lokalisierten als bei der generalisierten Form der Erkrankung auf.

C haben eine Spezifität von $46 \%$ für die Erkrankung.

D sind häufig gegen die Proteinase 3 gerichtet.

E sind für die Therapiewahl entscheidend.

5 Welche Aussage ist falsch?

Das Churg-Strauss-Syndrom zeigt

A histologisch eine nicht-nekrotisierende Vaskulitis.

B in den befallenen Geweben eine Eosinophilie.

C häufig eine Koinzidenz mit dem Asthma bronchiale.

D ANCA mit Spezifität gegen Myeloperoxidase.

E vorwiegend einen Befall der kleinen Gefäße.
6 Welche Aussage ist falsch?

Bei der Wegener'schen Granulomatose liegt ein erhöhtes Mortalitätsrisiko vor bei

A einem Alter $>65$ Jahre.

B einer Beteiligung im Hals-Nasen-Ohrenbereich.

C kardialen Symptomen durch das Churg-Strauss-Syndrom.

D einer gastrointestinalen Beteiligung.

E einem Serumkreatinin $\geq 150 \mu \mathrm{mol} / \mathrm{L}$

7 Welche Aussage ist richtig?

Bronchozentrische Granulome treten auf bei

A der rheumatoiden Arthritis.

B pulmonalen mykobakteriellen Infektionen.

C der Wegener'schen Granulomatose.

D der ankylosierenden Spondylitis.

E der nekrotisierenden sarkoiden Granulomatose.

8 Welche Aussage ist falsch?

Eine alveoläre Hämorrhagie tritt auf bei

A der lymphoiden Granulomatose.

B dem Goodpasture Syndrom.

C der Wegener'schen Granulomatose.

D dem Churg-Strauss-Syndrom.

E idiopathisch.

9 Welche Aussage ist falsch? Die bronchozentrische Granulomatose

A tritt koinzident mit einem Asthma bronchiale im Rahmen einer allergischen bronchopulmonalen Aspergillose auf.

B hat eine gutartige Prognose.

C ist mit dem histologischen Befund bronchozentrischer Granulome gesichert.

D tritt meist zwischen dem 4. und 7. Lebensjahrzehnt auf.

E kann mit einem oralen Kortikoid behandelt werden.

10 Welche Aussage ist falsch?

Kriterien des American College of Rheumatology für

das Churg-Strauss-Syndrom sind

A Mono- oder Polyneuropathie.

B Sinusitis.

C Asthma bronchiale.

D Kavernen, Infiltrate oder Granulome im Röntgen.

E histologisch extravaskuläre Eosinophilie. 